\title{
Circulating miRNA-24 and its target YKL-40 as potential biomarkers in patients with coronary heart disease and type 2 diabetes mellitus
}

\author{
Xin Deng ${ }^{1,2, *}$, Yaofang Liu ${ }^{3, *}$, Mao Luo ${ }^{1,2}$, Jian Wu ${ }^{4}$, Rongyue $\mathrm{Ma}^{5}$, Qin Wan ${ }^{6}$ and \\ Jianbo $\mathbf{W} \mathbf{u}^{1,2}$ \\ ${ }^{1}$ Drug Discovery Research Center, Southwest Medical University, Luzhou, Sichuan, China \\ ${ }^{2}$ Laboratory for Cardiovascular Pharmacology of Department of Pharmacology, The School of Pharmacy, Southwest Medical \\ University, Luzhou, Sichuan, China \\ ${ }^{3}$ Department of Gynaecology and Obstetrics, The Affiliated Hospital of Southwest Medical University, Luzhou, Sichuan, China \\ ${ }^{4}$ Medical Research Center, The Affiliated Hospital of Southwest Medical University, Luzhou, Sichuan, China \\ ${ }^{5}$ The Lee Woo Sing College, Chinese University of Hong Kong, Sha Tin, Hong Kong \\ ${ }^{6}$ Department of Endocrinology, The Affiliated Hospital of Southwest Medical University, Luzhou, Sichuan, China \\ *Xin Deng and Yaofang Liu contributed equally to this work \\ Correspondence to: Jianbo Wu, email: jbwucn1996@yahoo.com \\ Qin Wan, email: wanqin3@163.com \\ Keywords: biomarker, circulating miR-24, coronary heart disease, YKL-40, type 2 diabetes \\ Received: January 19, $2017 \quad$ Accepted: May 23, $2017 \quad$ Published: June 21, 2017 \\ Copyright: Deng et al. This is an open-access article distributed under the terms of the Creative Commons Attribution License 3.0 \\ (CC BY 3.0), which permits unrestricted use, distribution, and reproduction in any medium, provided the original author and source \\ are credited.
}

\section{ABSTRACT}

Type 2 diabetes mellitus (DM2) is associated with cardiovascular complications and is characterized by high levels of YKL-40, an inflammatory glycoprotein involved in endothelial dysfunction. We investigated the predictive potential of circulating miR24 in coronary heart diseases (CHD) DM2 patients with CHD, and control subjects. Blood samples were taken from 94 subjects of both genders, and divided over three groups as follows; patients with CHD, patients with DM2 and CHD, and control subjects. Both miR-24 (using real time PCR) and routine parameters were measured. Using bioinformatic analysis and luciferase assays, we found that miR-24 has high complementarity and a high degree of species conservation with respect to the binding sites within the 3' UTR of the YKL-40 mRNA. The expression levels of circulating miR-24, determined by quantitative real time PCR, were significantly decreased in peripheral blood of DM2-CHD and CHD patients compared with controls. Furthermore, miR-24 strongly associated with DM2-CHD, negatively correlated with YKL-40 in DM2CHD and DM2 patients after conducting multiple regression analysis. These results provide a novel regulatory mechanism of circulating miR-24 in regulating YKL-40 levels in DM2-CHD, may serve as a biomarker for predicting patients with DM2 and CHD.

\section{INTRODUCTION}

Coronary heart disease (CHD) is a major cause of morbidity and mortality among patients with diabetes mellitus. DM2 is characterized by systemic insulin resistance, which promotes hyperglycemia [1], and it has been proposed that these metabolic abnormalities directly increases the risk of cardiovascular diseases. Endothelial dysfunction plays a key role in determining myocardial infarction in all clinical manifestations of ischemic heart diseases [2,3]. Previous studies demonstrated that endothelial dysfunction has been linked to DM2 and insulin resistance [4].

YKL-40, also named chitinase 3-like 1 (Chi311) $[5,6]$, produced by a number of different cell types, 
including: cancer cells, macrophages, neutrophils [7-10], is an inflammatory glycoprotein involved in endothelial dysfunction by regulating chemotaxis, cell attachment and migration, reorganization as a response to endothelial injury [11]. YKL-40 was found to induce coordination of membrane-bound receptor syndecan-1 and integrin $\alpha v \beta 3$ and to activate an intracellular signaling cascade, including focal adhesion kinase and MAPK ERK1/2 in endothelial cells [12]. Several studies demonstrated that elevated serum YKL-levels are associated with the presence of coronary artery disease and myocardial infarction [13]. Moreover, elevated serum YKL-40 levels are elevated both in patients with type 1 and type 2 diabetes $[14,15]$.

Recent studies have shown that miRNA expression profiles can contribute to the development of DM2 and its complications [16]. Circulating miRNAs levels are strongly associated with endothelium activation or damage $[17,18]$, suggesting that the modification of miRNAs can be developed as novel disease diagnostic or prognostic biomarkers at an early stage in CHD. miR-24 belongs to miR-23 27 24 cluster, and is highly expressed in endothelial cells [19]. During the development of cardiovascular disease, miR-24 plays an important role in regulating endothelial function, such as proliferation, apoptosis, angiopoiesis, inflammation, and differentiation
[20]. miR-24 also modulated the TGF- $\beta$ signaling pathway through targeting gene FURIN [21]. However, a relationship between miR-24 and the YKL-40 signaling pathway has not been reported. The current study was carried out to evaluate the change of miR-24 in CHD subjects, and DM2-CHD subjects compared to control ones. In addition, we investigated the correlation between both miR-24 and YKL-40, and the utility of circulating miR-24 as a potential biomarker for predicting DM2CHD.

\section{RESULTS}

\section{Clinical data}

The study consisted of three subject groups: CHD patients $(n=35), D M 2$ patients with $\operatorname{CHD}(n=28)$ and control subjects $(n=31)$. The clinical data of the three subject groups are shown in Supplemental Table 1. In the all subject groups, there was no statistically significant difference in the sex distribution $(p>0.05)$, whereas blood glucose level differed significantly between DM2 patients with CHD compared with $\mathrm{CHD}$ patients and controls $(p<0.05)$. There were no significant differences in liver function, including alanine transaminase (ALT),

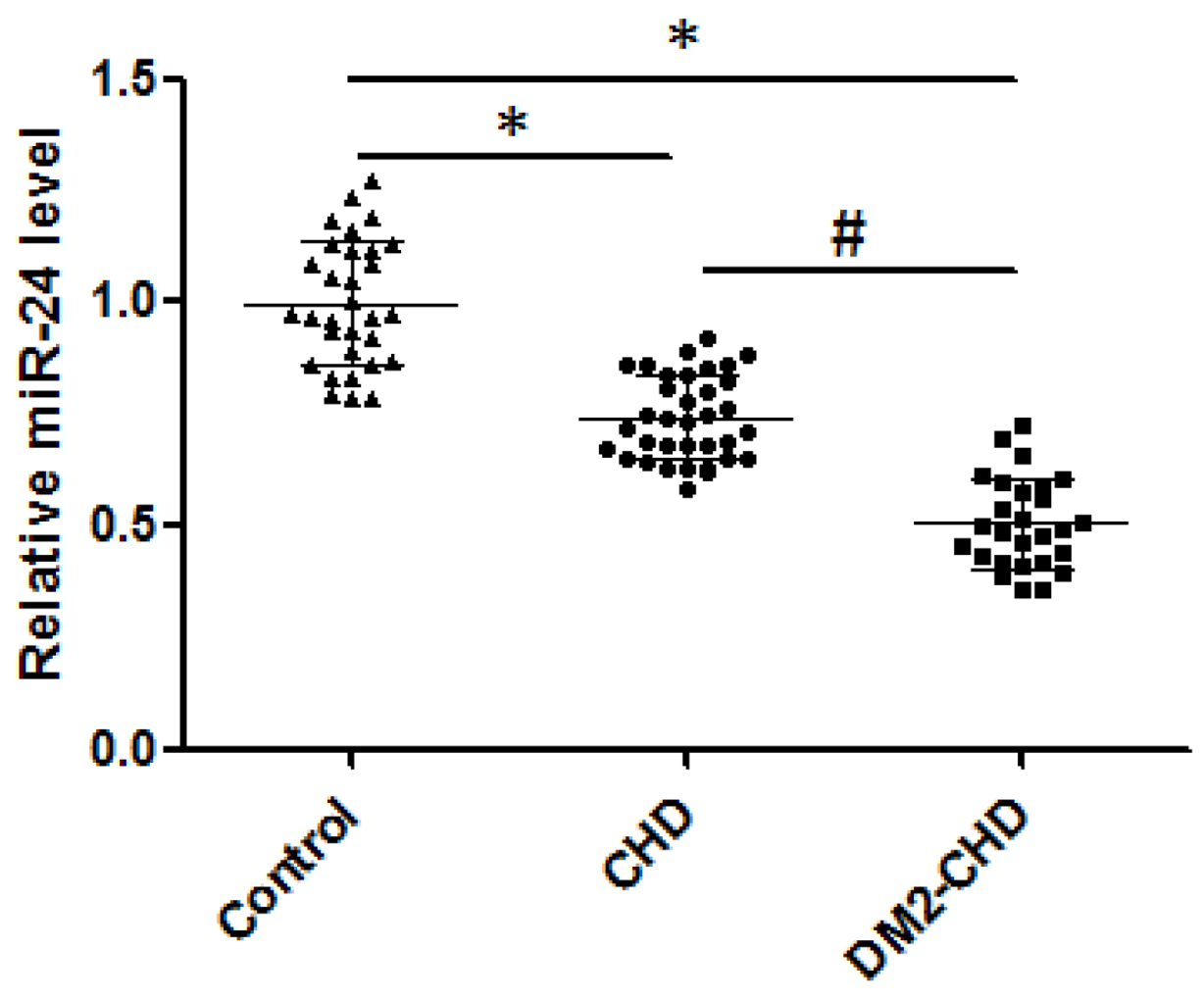

Figure 1: The levels of circulating miR-24. Expression of miR-24 mRNA level was confirmed through qRT-PCR analysis in control, CHD, and DM2-CHD, respectively. The products of qRT-PCR were detected by agarose gel electrophoresis. Total RNA extraction was performed using TRIzol Reagent. The data were normalized to U6 RNA in each sample. The qRT-PCR data were analyzed using the $2^{-\Delta \Delta C t}$ method. ${ }^{*} P<0.05$, Control vs CHD, DM2-CHD; ${ }^{*} P<0.05$ vs CHD group. 
aspartate transaminase (AST) between DM2 patients with CHD compared with CHD patients without chronic complications and controls. Furthermore, Gensini score was significantly higher in DM2 patients with CHD compared to CHD patients and controls $(p<0.05)$. There

A

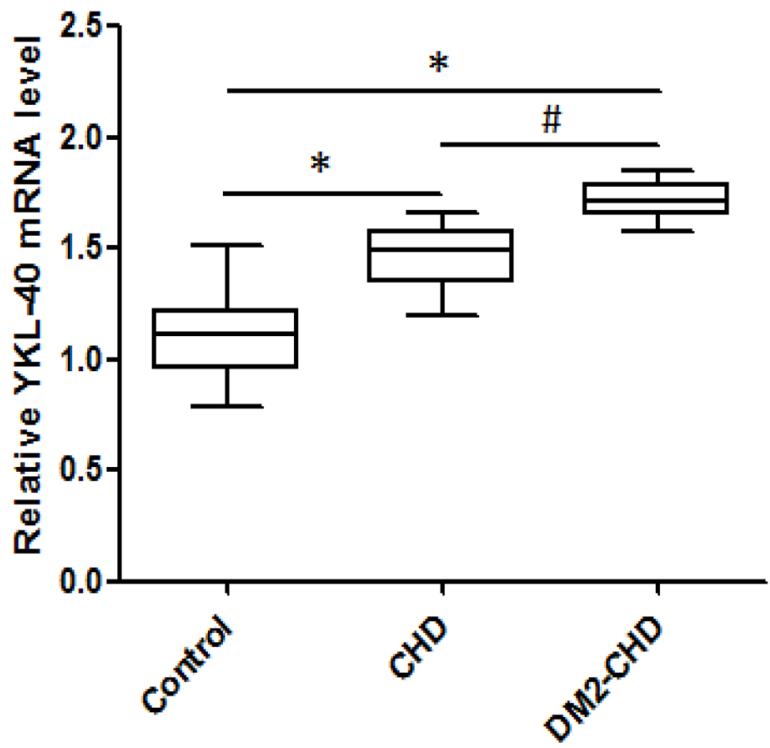

was also significant difference in Gensini score between CHD patients and those with control subjects $(p<0.05)$. For serum lipids, low-density lipoprotein (LDL), highdensity lipoprotein (HDL), total cholesterol (TC), and triglyceride (TG) were no significant difference between

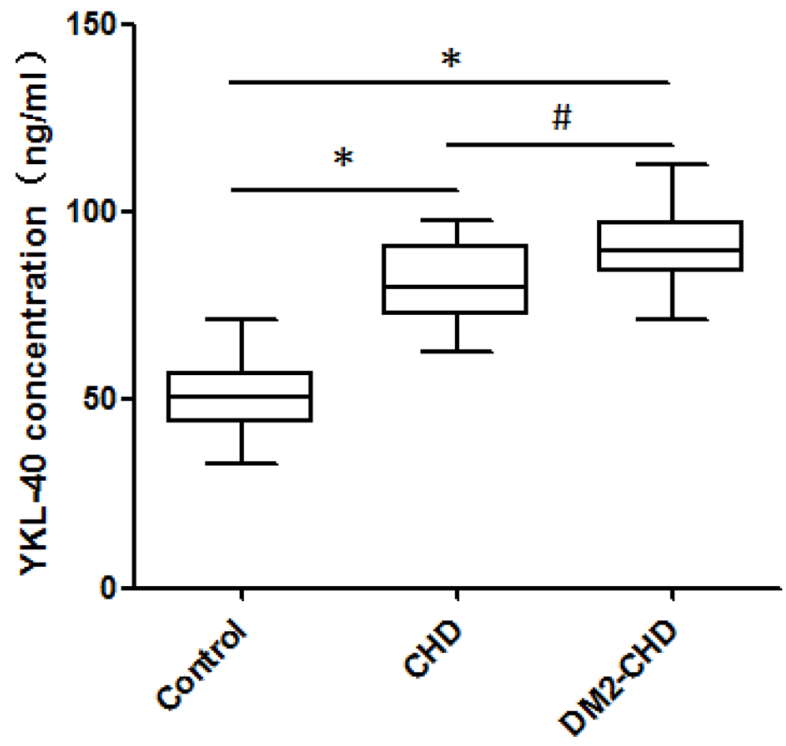

Figure 2: The expression level of circulating YKL-40. (A) Expression of YKL-40 mRNA level was confirmed through qRTPCR analysis in control, CHD, and DM2-CHD, respectively. Total RNA extraction was performed using TRIzol Reagent. The data were normalized to $18 \mathrm{~S}$ rRNA in each sample. The qRT-PCR data were analyzed using the $2^{-\triangle \Delta C t}$ method. (B) YKL-40 levels were determined using ELISA following a BCA assay for PPP (platelet poor plasma) in control, CHD, and DM2-CHD, respectively. ${ }^{*} P<0.05$, Control $v s$ CHD, DM2-CHD; ${ }^{*} P<0.05$ vs CHD group.
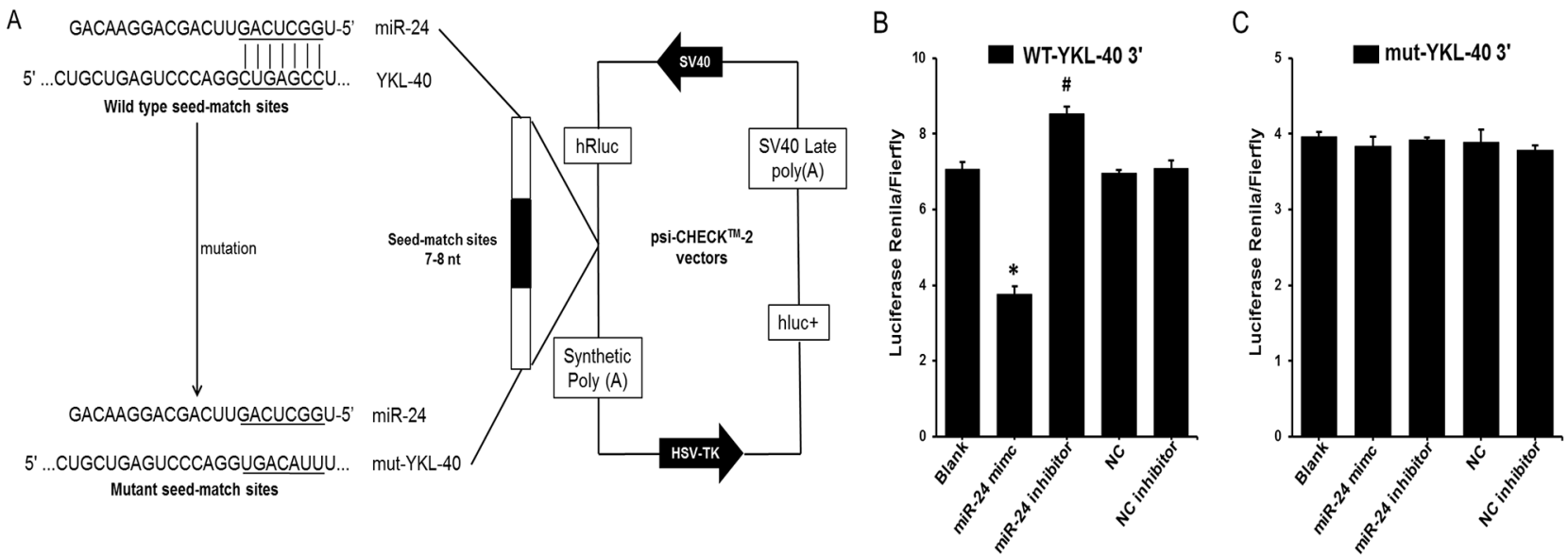

Figure 3: YKL-40 is a direct target of miR-24. (A) Schematic representation of the YKL-40 3' UTR luciferase reporter plasmid. The "seed sequences" and the point mutations in the seed sequences are underlined. (B) and (C) A miR-24 mimic (50 nmol/L), miR-24 inhibitor $(50 \mathrm{nmol} / \mathrm{L})$ or control oligo $(50 \mathrm{nmol} / \mathrm{L})$ was co-transfected with the psi-CHECK-2 wild-type or mutated YKL-40 3' UTR sequence vectors in HEK 293 cells. The relative luciferase activity is reported. All data are presented as the mean \pm SEM of triplicate independent experiments. ${ }^{*} p<0.05$, miR-24 mimic experimental $v s$. Blank, miR-24 inhibitor experimental, NC and NC inhibitor. ${ }^{*} P<0.05$, miR-24 inhibitor experimental vs. Blank, miR-24 mimic experimental, NC and NC inhibitor. 
DM2 patients with CHD compared with CHD patients and controls. In addition, no significant differences were found in renal function parameters, including serum creatinine (Cr.), blood urea nitrogen (BUN), and uric acid (UA) between DM2 patients with CHD compared with CHD patients and controls.

\section{Expression levels of circulating miR-24 in DM2 patients with $\mathrm{CHD}, \mathrm{CHD}$ patients and control subjects}

The levels of circulating miR-24 were significantly lower in CHD patients than that in control subjects $(0.741 \pm 0.094$ vs. $0.998 \pm 0.140 ; \mathrm{p}<0.05)$. In addition, circulating levels of miR-24 were remarkably decreased in DM2 patients with CHD compared with controls $(0.506 \pm 0.0101$ vs. $0.998 \pm 0.140 ; p<0.05)$ (Figure 1$)$.

A

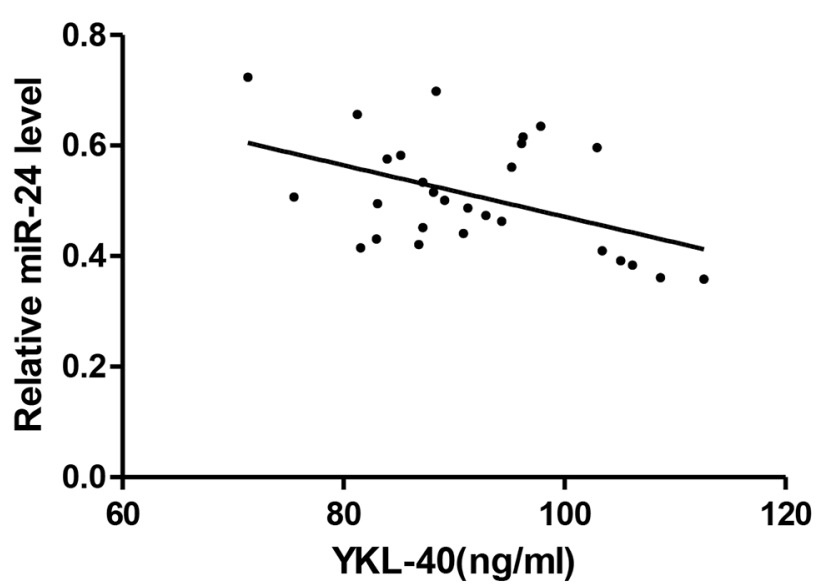

C

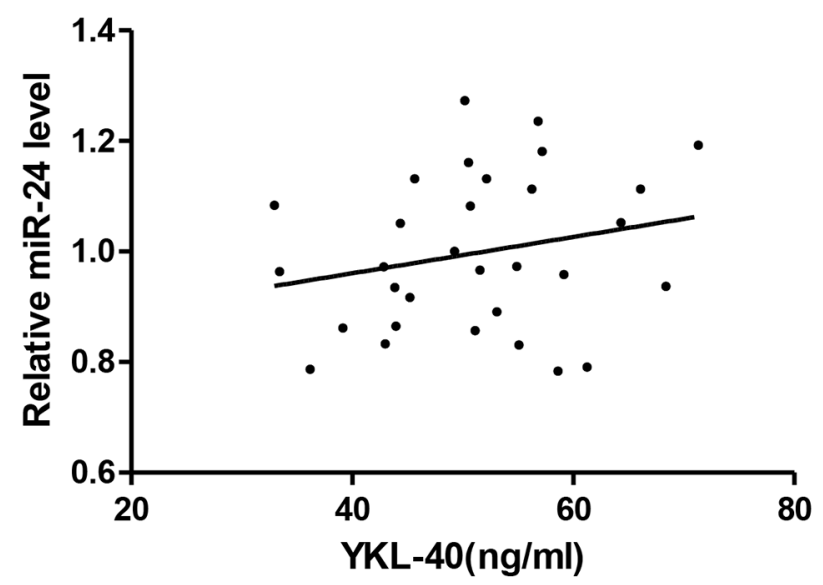

\section{Expression level of circulating YKL-40}

YKL-40 mRNA levels were analyzed by qRTPCR and compared with the level of endogenous genes $18 \mathrm{~S}$ rRNA. As shown in Figure 2A, the YKL-40 mRNA expression levels were up-regulated and significantly greater in both DM2-CHD and CHD subjects compared with controls, moreover, the levels of YKL-40 mRNA expression were significantly higher in DM2-CHD patients than that in CHD subjects $(1.716 \pm 0.083$ vs. $1.47 \pm 0.132 ; \mathrm{p}<0.05)$. Furthermore, we estimated the total amount of circulating YKL-40 protein antigen by ELISA. As shown in Figure 2B, the average amount of YKL-40 antigen were significantly increased in both DM2-CHD and CHD subjects compared with controls. Higher levels of YKL-40 protein were also found in DM2-CHD patients compared with CHD subjects $(91.6 \pm 10.0$ vs. $80.5 \pm 10.6$;

B

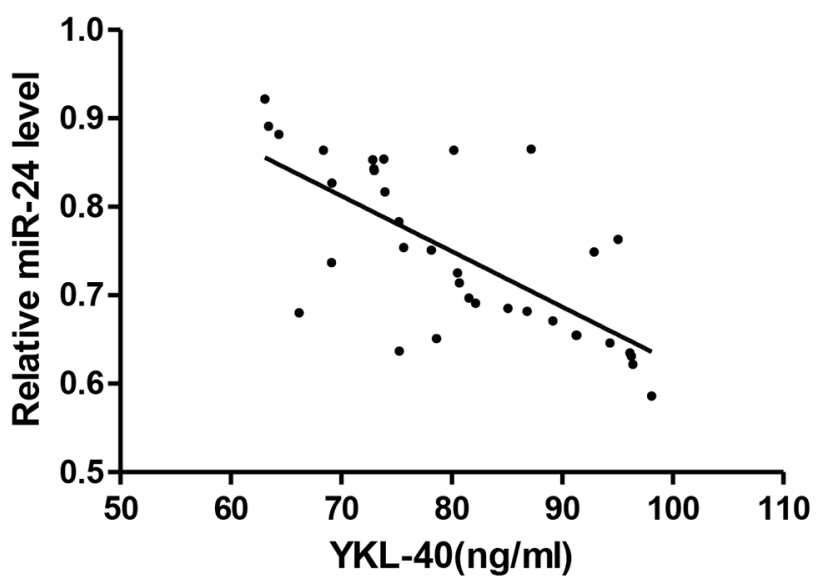

Figure 4: Elevated YKL-40 and reduced miR-24 identified in DM2-CHD, CHD, and control. Correlation between circulating miR-24 and plasma YKL-40 in DM2-CHD (A), CHD (B), and controls (C), respectively. 
$\mathrm{p}<0.05)$. These results suggested there being increased changes in YKL-40 levels in both DM2-CHD and CHD.

\section{Prediction analysis}

Predictions of hsa-miR-24 target genes were performed using a bioinformatics-based approach with Targetscan human software [22], and further confirmed by using the more efficient software miRanda [23], MicroRNA.org [24] and Microcosm [25]. According to the bioinformatics predictions, the software provided information regarding target site accessibility. As shown in Figure 3, there is only a single predicted miR-24 target site in the YKL-40 mRNA 3' UTR based on good complementarity $\left(\Delta \mathrm{G}^{\circ} \sim-25.67 \mathrm{kcal} / \mathrm{mol}\right)$, indicating a high degree of site conservation among different mammalian species.

\section{YKL-40 is a direct target of miR-24}

To investigate the predicted interaction of miR24 with YKL-40 mRNA, the 3' UTR of human YKL-40 containing the putative miR-24 binding sites was cloned into the psi-CHECK2TM vector downstream of the Renilla luciferase coding sequence and co-transfected with miR-24 mimic, inhibitor or control oligo into HEK 293 cells. An empty vector was used as control (Figure 3A). In the presence of the YKL 3' UTR, the miR-24 mimic significantly decreased the relative luciferase activity to approximately $50 \%$ compared to co-transfection with

\section{A}

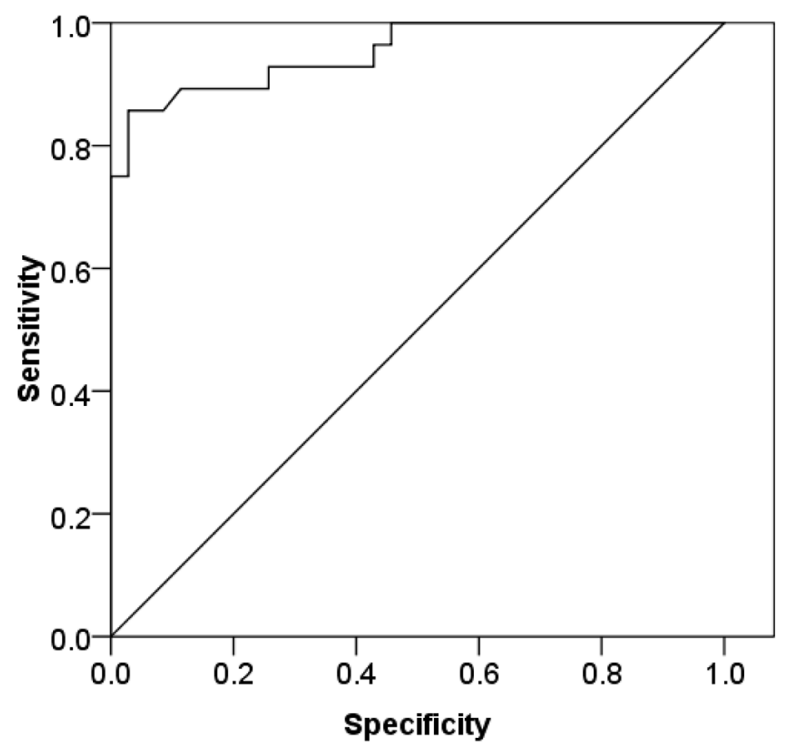

miR-NC. The relative luciferase activity was significantly increased by the miR-24 inhibitor (Figure 3B). Furthermore, to investigate whether the predicted miR-24 binding sites mediate the effect on YKL-40, miR-24 seed sequences binding to the YKL-40 mRNA 3' UTR were mutated (Figure 3C). The effects of the miR-24 mimic and inhibitor were abrogated compared to co-transfection of control oligo with vector or empty vector (Figure 3C), suggesting that miR-24 could directly target YKL-40 3' UTRs.

\section{Correlation analysis}

Pearson's coefficient correlation was performed to evaluate the association of circulating miR-24 with YKL-40 level in DM2 patients with CHD, CHD patients and controls, respectively. As shown in Figure 4A-4C, miR-24 was significantly and negatively correlated with YKL-40 in either DM2 patients with CHD $(r=-0.508$, $\mathrm{p}<0.01)$ or CHD patients $(r=-0.703, \mathrm{p}<0.001)$. When these parameters were compared in the control subjects, miR-24 was not significantly correlated with YKL-40 ( $r$ $=0.227, \mathrm{p}>0.05)$.

\section{Evaluation of the clinical biomarker}

To evaluate if circulating miR-24 could significantly differentiate between DM2 patients with CHD and CHD patients from control subjects. The analysis of ROC curves were constructed and area under the curves (AUCs) were

B

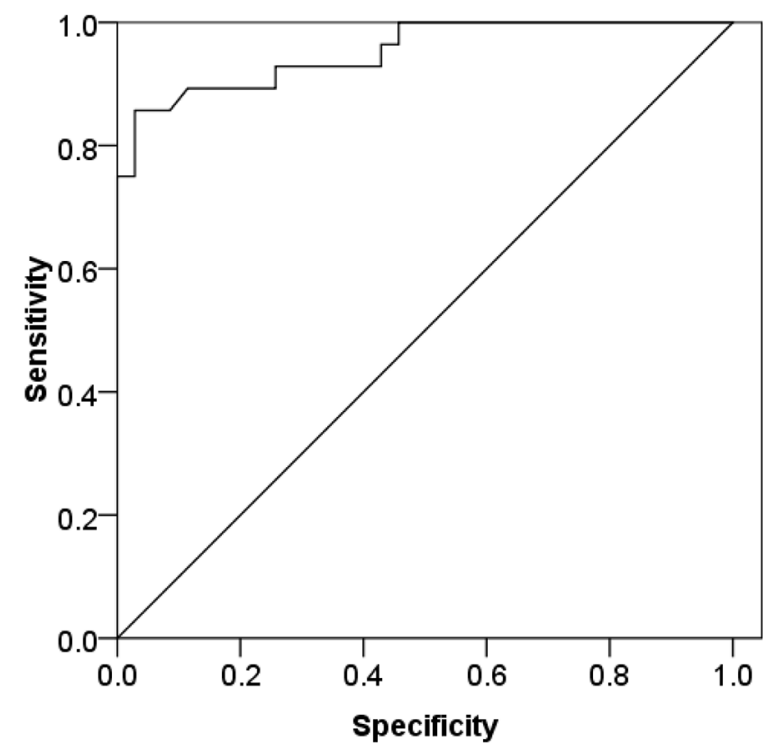

Figure 5: The biomarker potential of circulating miR-24 for DM2-CHD, CHD, and control. ROC analysis was used to evaluate the ability of circulating miR-24 to distinguish between different groups. (A) MiR-24 distinguished DM2-CHD patients from controls with area under curve (AUC) of 0.975 (Confidence interval [IC]: 0.944 1.0; $<<0.001$ ). (B) MiR-24 distinguished DM2-CHD patients from CHD patients (AUC: 0.953; CI: 0.899 1.0; $<<0.001$ ). 
calculated. The ROC curve of miR-24 showed significant ability to discriminate DM2-CHD from CHD patients and control subjects with an AUC of 0.975 (CI: 0.944 1.0; $\mathrm{P}<0.001$ ), (Figure 5A). MiR-24 also discriminated DM2CHD patients from CHD with an AUC of 0.953 (CI: 0.899 $\sim 1.0 ; \mathrm{P}<0.001$ ) (Figure 5B).

\section{DISCUSSION}

In the current study, we reported a significant reduction in the expression levels of circulating miR24 in DM2 patients with CHD and CHD patients in comparison to control subjects. These results were further confirmed in multivariate logistic regression analysis which revealed strong association of miR-24 expression with DM2-CHD. We also showed that circulating miR-24 was significantly and negatively correlated with YKL-40 in DM2-CHD, as determined using Pearson's correlation analysis. The findings in our study were that circulating miR-24 discriminated between DM2 patients with CHD, CHD patients, and control subjects, as assessed by ROC analysis.

Circulating YKL-40 levels are elevated both in patients with type 1 and type 2 diabetes, known to be at high risk for the development of cardiovascular diseases and insulin resistance [26]. Several studies demonstrate, that elevated circulating YKL-40 levels are independently associated with the presence and extent of CHD. However, no mechanistic analyses were reported. We now provide that circulating YKL-40 is coordinately regulated by miR24 in DM2 patients with CHD.

Previous study demonstrated that miRNAs have been reported to regulate vascularity after myocardial infarction [20]. MiRNA profiling has revealed that miR24 levels were noted to be reduced in DM2 [27]. An increasing number of studies supporting the pivotal role of circulating miRNAs in diabetic patients show that circulating miRNAs may be considered novel biomarkers of cardiovascular diseases [28]. Through bioinformatic analyzing and luciferase assays, we determined miR-24 as directly targeting the 3 'UTR of the YKL-40 gene. miR24 is highly expressed in endothelial cells. We found that miR-24 strongly associated with DM2-CHD, negatively correlated with YKL-40 in DM2-CHD and CHD patients after conducting multiple regression analysis. Our findings lend insight into miR-24-based regulation of YKL-40 and provide a mechanism for the high circulating YKL-40 levels and cardiovascular events observed with DM2.

CHD is responsible for the main proportion of mortality associated with DM2. miR-24 plays an important role in the development of cardiovascular events. While conflicting results have been published regarding the impact of miR-24 on mouse myocardial infarction models $[20,29,30]$. More recently, miR-24 was reported to upregulate vascular endothelial cells von Willebrand factor (VWF) in diabetic patients and correlate with thrombotic event [31]. In line with this study, we identified that decreased miR-24 in plasma is correlated with increased YKL-40 level in DM2 patients with CHD.

Another novel finding, in our results, circulating miR-24 was able to discriminate between DM2 patients with CHD from CHD patients and control subjects with an area under the curve (AUC) of the ROC of 0.975 (CI: 0.944 1.0, $\mathrm{P}<0.001$ ), suggested that circulating miR-24 exhibited not only a potential prognostic value but also a biomarker for predicting DM2 patients with CHD. We demonstrated that decreased levels of miR-24 is associated with elevated levels of YKL-40 in DM2 patients with CHD.Furthermore, we described a novel regulatory mechanism of miR-24 regulating conserved target YKL-40 expression by directly binding to the YKL-40 mRNA 3' UTR seed sequence. Our study is to evaluate the possibility of peripheral blood miR-24 as a potential biomarker for DM2 patients with CHD. However, in the present study, the biomarker potential of circulating miR-24 for DM2 patients with CHD was conducted in a relatively small sample size of cases and controls, and thus the utility of miR-24 biomarker will need further evaluation in a large clinical study in future.

\section{MATERIALS AND METHODS}

\section{Patient selection}

All patients provided written informed consent 94 research subjects were Han Chinese individuals who received oral glucose tolerance test (OGTT) and other medical health checkups. Coronary heart disease, in particular acute myocardial infarction (AMI), is the major cause of morbidity and mortality in adults. The patients included in this study sustained an initial CHD event (AMI), other types not included in the group, such as coronary artery bypass graft, percutaneous transluminal angiography or angiographically validated CHD. This study comprised three groups, including 31 control subjects, 35 CHD subjects, and 28 DM-CHD subjects. AMI diagnosis was based on following criteria: (1) chest pain lasting $>20$ minutes, (2) pathological $\mathrm{Q}$ waves or ST-segment elevation/depression on the ECG, and (3) elevation of traditional myocardial markers. All research subjects were received between September 20 and January 20, 2016. Control subjects were not allowed to take aspirin, atorvastatin or nonsteroidal anti-inflammatory drugs for 10 days prior to the investigation. The patient study was conducted in accordance with the Declaration of Helsinki, and the study protocol was approved by the Ethics Committee of Affiliated Hospital of Southwest Medical University. The diagnosis of DM2 was performed according to the World Health Organization criteria, i.e., when fasting, glucose was $\geq 7 \mathrm{mmol} / \mathrm{L}(126 \mathrm{mg} / \mathrm{dL})$, the 
two hour oral glucose tolerance test glucose level was $\geq 11.1 \mathrm{mmol} / \mathrm{L}(200 \mathrm{mg} / \mathrm{dL})$ or the subjects had a clinical diagnosis of the disease.

\section{Bioinformatic analysis}

We searched for putative miRNAs that target the 3' UTR of the YKL-40 mRNA using Targetscan human software 32. The results were confirmed using different types of software, including miRanda33, MicroRNA. org34 and Microcosm35. The evolutionary conservation was studied using Multiple EM for Motif Elicitation (MEME) software (http://meme.nbcr.net/meme/tools/ meme).

\section{Isolation of total RNA and miRNA}

Total RNA extraction from blood was performed with TRIzol (Invitrogen, Carlsbad, CA.). Small RNAs, including miRNAs by using the mirVanaTM miRNA Isolation Kit (Ambion, Austin, TX) according to the manufacturer's recommendations. The eluted small RNA was stored at $-80^{\circ} \mathrm{C}$.

\section{3'UTR luciferase reporter gene assay}

3'UTR luciferase reporter geneassay was studied as described [32]. A fragment of the YKL-40 mRNA 3' UTR containing the putative or mutated miR-24 binding site was amplified by RT-PCR from MEG-01 cell total RNA. The products were inserted into the XhoI and NotI restriction sites of thepsi-CHECK2TM vectors (Promega) downstream from the Renilla luciferase coding sequence. The constructs were cotransfected with miR-24 mimic, inhibitor and control oligo into HEK 293 cells using Lipofectamine 2000 (Invitrogen, Grand Island, NY). Cells were harvested after 48 hours of transfection, and firefly luciferase activity was measured using the DualLuciferase Reporter Assay System Kit (Promega, E1910) and the luminometer Orion II (Berthold Detection Systems, Germany).

\section{miR-24 and its target gene YKL-40 quantitative RT-PCR assay}

Validation of the expression of the sequencespecific miR-24 was determined using quantitative stemloop qRT-PCR. Validation of its target gene YKL-40 was performed using qRT-PCR. U6 and 18S rRNA were used as internal controls for normalization. Briefly, small RNAs were transcribed into cDNA using the designed miRNA specific stem loop-RT primers and the NCode ${ }^{\mathrm{TM}}$ miRNA First-Strand cDNA Synthesis Kit (Invitrogen). Then, 500 ng of total RNA was reverse transcribed into cDNA using the M-MLV Reverse Transcription Kit (Promega, USA). All qPCRs (20 $\mu$ l total reaction) were performed with 5 $\mu \mathrm{L}$ of generated cDNA using the SYBR Green Master
Mix protocol (TaKaRa Biotechnology Co., Ltd) with the ABI PRISM 7500 system (Applied Biosystems, Forster City, CA, USA). The reactions were incubated in a 96-well plate at $95^{\circ} \mathrm{C}$ for $10 \mathrm{~min}$, followed by 40 cycles of $95^{\circ} \mathrm{C}$ for $15 \mathrm{~s}, 60^{\circ} \mathrm{C}$ for $30 \mathrm{sec}, 72^{\circ} \mathrm{C}$ for $30 \mathrm{sec}$. The dissociation curve analysis of the PCR products was determined in the final stage of $55^{\circ} \mathrm{C}$ to $95^{\circ} \mathrm{C}$. All reactions were run in triplicate, and each sample was replicated three times. All fold changes using qRT-PCR were determined using the $2^{-\triangle \Delta C T}$ mean \pm SEM $[32,33]$. All primers are listed in Supplementary Table 2.

\section{Measurement of YKL-40}

Blood was collected into citrate anticoagulant, and plasma were prepared by centrifugation. YKL-40 antigen was measured using the human YKL-40 total antigen assay ELISA kit (R\&D systems, Minneapolis, MN). The detection limit was $15 \mu \mathrm{g} / \mathrm{L}$. The intra-assay coefficient of variation $(\mathrm{CV})$ was $<5 \%$ and the inter-assay $\mathrm{CV}$ was $<6 \%$. All samples from each patient were analyzed on the same plate to reduce inter-assay $\mathrm{CV}$.

\section{Statistical analysis}

Results were calculated as mean \pm standard error of mean $(\mathrm{M} \pm \mathrm{SEM})$. Pearson $\chi 2$ test was used to compare qualitative variables represented as cut off values. Statistical analyses were done using SPSS version 20.0. Multivariate logistic regression analysis was done to determine the variables that independently contributed to the presence of CHD. Discriminating between patients and controls was identified using receiver-operating characteristic (ROC) analysis and area under the curve (AUC) was calculated for circulating miR-24 to assess predictive value. $\mathrm{P}<0.05$, were considered to be significant.

\section{CONFLICTS OF INTEREST}

The authors have no financial interest in this project.

\section{CONSENT FOR PUBLICATION}

Consent for publication was obtained from the patients and controls as part of the consent to participate.

\section{ETHICS APPROVAL AND CONSENT TO PARTICIPATE}

Patients were selected from the study entitled "Circulating miRNA-24 and its target YKL-40 as potential biomarkers in patients with coronary heart disease and type 2 diabetes mellitus". The Southwest Medical University ethics committee approved the study. All subjects provided written consent. 


\section{FUNDING}

This work was supported by the National Natural Science Foundation of China Grant (81570263, JW), Grant of Sichuan Province Science and Technology Agency Grant (2014FZ0104, JW; 2016RZ0076, XD), and a Ministry of Science and Technology of China (016YFC0901205, QW).

\section{REFERENCES}

1. Eckel RH, Wassef M, Chait A, Sobel B, Barrett E, King G, Lopes-Virella M, Reusch J, Ruderman N, Steiner G, Vlassara H. Prevention conference VI: Diabetes and cardiovascular disease: writing Group II: pathogenesis of atherosclerosis in diabetes. Circulation. 2002;105:e138-43.

2. Summers MR, Lerman A, Lennon RJ, Rihal CS, Prasad A. Myocardial ischaemia in patients with coronary endothelial dysfunction: insights from body surface ECG mapping and implications for invasive evaluation of chronic chest pain. Eur Heart J. 2011;32:2758-65.

3. Zeiher AM, Drexler H, Wollschlager H, Just H. Endothelial dysfunction of the coronary microvasculature is associated with coronary blood flow regulation in patients with early atherosclerosis. Circulation. 1991;84:1984-92.

4. Creager MA, Luscher TF, Cosentino F, Beckman JA. Diabetes and vascular disease: pathophysiology, clinical consequences, and medical therapy: Part I. Circulation. 2003; 108:1527-32.

5. Hakala BE, White C, Recklies AD. Human cartilage gp39 , a major secretory product of articular chondrocytes and synovial cells, is a mammalian member of a chitinase protein family. J Biol Chem. 1993;268:25803-10.

6. Nyirkos P, Golds EE. Human synovial cells secrete a $39 \mathrm{kda}$ protein similar to a bovine mammary protein expressed during the non-lactating period. Biochem J. 1990;269:265- 68.

7. Rehli M, Krause SW, Andreesen R. Molecular characterization of the gene for human cartilage gp-39 (CHI3L1), a member of the chitinase protein family and marker for late stages of macrophage differentiation. Genomics. 1997;43:221-225.

8. Volck B, Price PA, Johansen JS, Sørensen O, Benfield TL, Nielsen HJ, Calafat J, Borregaard N. YKL-40, a mammalian member of the chitinase family, is a matrix protein of specific granules in human neutrophils. Proc Assoc Am Physicians. 1998;110:351-60.

9. Boot RG, van Achterberg TA, van Aken BE, Renkema GH, Jacobs MJ, Aerts JM, de Vries CJ. Strong induction of members of the chitinase family of proteins in atherosclerosis: chitotriosidase and human cartilage gp-39 expressed in lesion macrophages. Arterioscler Thromb Vasc Biol. 1999; 19:687-94.

10. Kzhyshkowska J, Yin S, Liu T, Riabov V, Mitrofanova I. Role of chitinase-like proteins in cancer. Biol Chem. 2016;397:231-47.
11. Rathcke CN, Vestergaard H. YKL-40 - an emerging biomarker in cardiovascular disease and diabetes. Cardiovasc Diabetol. 2009;8:61.

12. Shao R, Hamel K, Petersen L, Cao QJ, Arenas RB, Bigelow C, Bentley B, Yan W. YKL-40, a secreted glycoprotein, promotes tumor angiogenesis. Oncogene. 28:4456-68.

13. Kastrup J, Johansen JS, Winkel P, Hansen JF, Hildebrandt P, Jensen GB, Jespersen CM, Kjoller E, Kolmos HJ, Lind I, Nielsen H, Gluud C; CLARICOR Trial Group. High serum YKL-40 concentration is associated with cardiovascular and all-cause mortality in patients with stable coronary artery disease. Eur Heart J. 2009;30:1066-72.

14. Nielsen AR, Erikstrup C, Johansen JS, Fischer CP, Plomgaard P, Krogh-Madsen R, Taudorf S, Lindegaard B, Pedersen BK. Plasma YKL-40: a BMI-independent marker of type 2 diabetes. Diabetes. 2008;57:3078-82.

15. Rathcke CN, Persson F, Tarnow L, Rossing P, Vestergaard H. YKL-40, a marker of inflammation and endothelial dysfunction, is elevated in patients with type 1 diabetes and increases with levels of albuminuria. Diabetes Care. 2009;32:323-28.

16. Caporali A, Meloni M, Völlenkle C, Bonci D, SalaNewby GB, Addis R, Spinetti G, Losa S, Masson R, Baker AH, Agami R, le Sage C, Condorelli G, et al. Deregulation of microRNA-503 contributes to diabetes mellitus-induced impairment of endothelialfunction and reparative angiogenesis after limb ischemia. Circulation. 2011;123:282-91.

17. Fichtlscherer S, Zeiher AM, Dimmeler S. Circulating microRNAs: biomarkers or mediators of cardiovascular diseases. Arterioscler Thromb Vasc Biol. 2011; 31:2383-90.

18. Tijsen AJ, Pinto YM, Creemers EE. Circulating microRNAs as diagnostic biomarkers for cardiovascular diseases. Am J Physiol Heart Circ Physiol. 2012; 303:H1085-95.

19. Zhou Q, Gallagher R, Ufret-Vincenty R, Li X, Olson EN, Wang $\mathrm{S}$. Regulation of angiogenesis and choroidal neovascularization by members of microRNA-23 27 24clusters. Proc Natl Acad Sci U S A. 2011;108:8287-92.

20. Fiedler J, Jazbutyte V, Kirchmaier BC, Gupta SK, Lorenzen J, Hartmann D, Galuppo P, Kneitz S, Pena JT, Sohn-Lee C, Loyer X, Soutschek J, Brand T, et al. MicroRNA-24 regulates vascularity after myocardial infarction. Circulation. 2011;124:720-30.

21. Wang J, Huang W, Xu R, Nie Y, Cao X, Meng J, Xu X, Hu $\mathrm{S}$, Zheng Z. MicroRNA-24 regulates cardiac fibrosis after myocardial infarction. J Cell Mol Med. 2012;16:2150-60.

22. Lewis BP, Burge CB, Bartel DP. Conserved seed pairing, often flanked by adenosines, indicates that thousands of human genes are microRNA targets. Cell. 2005; 120: 15-0.

23. John B, Enright AJ, Aravin A, Tuschl T, Sander C, Marks DS. Human MicroRNA targets. PLoS Biol. 2004; 2: e363.

24. Betel D, Wilson M, Gabow A, Marks DS, Sander C. The microRNA.org resource: targets and expression. Nucleic Acids Res. 2008; 36: D149-53. 
25. Griffiths-Jones S, Grocock RJ, van Dongen S, Bateman A, Enright AJ. miRBase: microRNA sequences, targets and gene nomenclature. Nucleic Acids Res. 2006; 34: D140-44.

26. Rathcke CN, Johansen JS, Vestergaard H. YKL-40, a biomarker of inflammation, is elevated in patients with type 2 diabetes and is related to insulin resistance. Inflamm Res. 2006, 55: 53-9.

27. Zampetaki A, Kiechl S, Drozdov I, Willeit P, Mayr U, Prokopi M, Mayr A, Weger S, Oberhollenzer F, Bonora E, Shah A, Willeit J, Mayr M. Plasma microRNA profiling reveals loss of endothelial miR-126 and other microRNAs in type 2 diabetes. Circ Res. 2010;107:810-17.

28. Guay C, Regazzi R. Circulating microRNAs as novel biomarkers for diabetes mellitus. Nat Rev Endocrinol. 2013;9:513-21.

29. Meloni M, Marchetti M, Garner K, Littlejohns B, SalaNewby G, Xenophontos N, Floris I, Suleiman MS, Madeddu P, Caporali A, Emanueli C. Local inhibition of
microRNA-24 improves reparative angiogenesis and left ventricle remodeling and function in mice with myocardial infarction. Mol Ther. 2013;21:1390-02.

30. Qian L, Van Laake LW, Huang Y, Liu S, Wendland MF, Srivastava D. miR-24 inhibits apoptosis and represses Bim in mouse cardiomyocytes. J Exp Med. 2011;208:549-60.

31. Xiang Y, Cheng J, Wang D, Hu X, Xie Y, Stitham J, Atteya G, Du J, Tang WH, Lee SH, Leslie K, Spollett G, Liu Z, et al. Hyperglycemia repression of miR-24 coordinately upregulates endothelial cell expression and secretion of von Willebrand factor. Blood. 2015;125:3377-87.

32. Luo M, Li R, Ren M, Chen N, Deng X, Tan X, Li Y, Zeng M, Yang Y, Wan Q, Wu J. Hyperglycaemia-induced reciprocal changes in miR-30c and PAI-1 expression in platelets. Sci Rep. 2016;6:36687.

33. Livak KJ, Schmittgen TD. Analysis of relative gene expression data using real-time quantitative PCR and the 2(-Delta Delta C(T)) Method. Methods. 2011; 25: 402-08. 